\title{
Novas ocorrências, novo sinônimo e uma lectotipificação em Loranthaceae
}

\author{
New occurrences, new synonym, and an lectotypification on Loranthaceae
}

\author{
Claudenir Simões Caires ${ }^{1,2}$
}

\begin{abstract}
Resumo
As novas ocorrências, Pusillanthus pubescens (Rizzini) Caires para Pernambuco e Struthanthus meridionalis Kuijt para o Mato Grosso, são apresentadas, bem como o registro de novas famílias de hospedeiros: Fabaceae, Malvaceae, Rutaceae e Solanaceae. Struthanthus polyanthus var. gracilis é proposto como sinônimo taxonômico para S. meridionalis. Um isótipo de Struthanthus pubescens, localizado no herbário do Field Museum, é escolhido como seu lectótipo.
\end{abstract}

Palavras-chave: Bolívia, Brasil, erva-de-passarinho, lectotipificação, novos hospedeiros.

\begin{abstract}
Two new records, Pusillanthus pubescens (Rizzini) Caires for the state of Pernambuco, and Struthanthus meridionalis Kuijt for the state of Mato Grosso, are presented, as well as records of new host families: Fabaceae, Malvaceae, Rutaceae and Solanaceae. Struthanthus polyanthus var. gracilis is proposed as a taxonomic synonym of S. meridionalis. An isotype of Struthanthus pubescens, located in the Field Museum herbarium, is chosen as its lectotype.
\end{abstract}

Key words: Bolivia, Brazil, mistletoe, lectotypification, new hosts.

\section{Introdução}

A família Loranthaceae é pantropical e possui 73 gêneros e ca. de 800 espécies de hemiparasitas, conhecidas nacionalmente como ervas-de-passarinho (Arruda et al. 2012). No Brasil a família é representada por 12 gêneros e ca. de 131 espécies distribuídas por todos os domínios fitogeográficos (Arruda et al. 2012).

O gênero Pusillanthus Kuijt é monotípico e foi recentemente descrito (Kuijt 2008) com base em Phthirusa trichodes Rizzini, registrada para a Venezuela (Rizzini 1975). Após quatro anos Caires et al. (2012) propuseram uma nova combinação, Pusillanthus pubescens (Rizzini) Caires, tendo como basiônimo Struthanthus pubescens Rizzini, descrita também para a Venezuela (Rizzini 1950).

O parátipo de S. pubescens (Pittier 13103) depositado no herbário do Missouri Botanical Garden foi lectotipificado por Caires et al. (2012) para representar o binômio da espécie, haja vista que o holótipo (Saer 472) depositado no herbário venezuelano (ver Rizzini 1950) e seus isótipos não foram localizados.

A rara ocorrência de Pusillanthus pubescens no Brasil, registrada por Kuijt (2008) e Rizzini $(1950,1975)$, foi alterada com as sinonimizações de Phthirusa caatingae Rizzini, Phthirusa trichodes Rizzini e Struthanthus pubescens var. bahiensis Rizzini propostas por Caires et al. (2012). Tais sinônimos e outras amostras oriundas de Alagoas e Paraíba ampliaram a ocorrência de Pusillanthus pubescens no Brasil (Caires et al. 2012).

Diversas mudanças nomenclaturais, resultantes de revisões morfológicas detalhadas, estão ocorrendo em Cladocolea Tiegh., Passovia Karst., Peristethium Tiegh., Phthirusa Mart. e Struthanthus Mart (Kuijt 2011, 2012, 2013).

Dentre estes gêneros, Struthanthus é o mais diverso possuindo ca. de 45 espécies (Kuijt 2013), e é frequentemente confundido com Passovia, sendo distinto deste por apresentar filetes delgados e íntegros com anteras dorsifixas (Kuijt 2011), evidentes nas flores masculinas.

\footnotetext{
${ }^{1}$ Universidade Estadual do Sudoeste da Bahia, Depto. Ciências Naturais, Estrada do Bem Querer, km 4, 45031-900, Vitória da Conquista, BA, Brasil.
}

${ }^{2}$ Email do autor: cscaires@hotmail.com 
Algumas espécies novas tem sido descritas em Struthanthus (Kuijt 2003, 2014a) e alterações surgem à medida que progridem novas investigações bibliográficas acompanhadas das análises de espécimes herborizados, como observado nos trabalhos de Caires et al. (2014), Dettke \& Waechter (2012) e Kuijt (2012, 2014b), porém a ausência de uma revisão genérica não permite estimar corretamente o seu quantitativo de espécies.

Os acervos dos herbários ASE*, F, IAN*, $\mathrm{K}, \mathrm{MG}^{*}, \mathrm{MO}, \mathrm{RB}^{*}, \mathrm{UB}^{*}$ e VEN (Thiers 2014), foram consultados, sendo aqueles com asterisco analisados pessoalmente, cujos exemplares estão aqui marcados com o sinal "!".

$\mathrm{O}$ avanço nas digitalizações dos acervos dos herbários internacionais e brasileiros, bem como a continuidade das visitas técnicas aos herbários nacionais e a incansável análise das imagens, nos trouxeram novidades sobre Pusillanthus e Struthanthus.

\section{Tratamento taxonômico}

Pusillanthus pubescens (Rizzini) Caires, Acta bot. bras. 26(3): 676, figs. 1-3. 2012.

Struthanthus pubescens Rizzini, Revista Brasil. Biol. 10(4): 404. 1950. - Tipo: Venezuela. Lara: Sanare, 1300 m, V.1930, fl., J. Saer 472 (holótipo VEN, não localizado, lectótipo aqui designado $\mathrm{F}$ [imagem!]).

Struthanthus pubescens var. bahiensis Rizzini, Revista Brasil. Biol. 10(4): 408. 1950. - Tipo: Brasil. Bahia: Jiquy, região Serra de Sincorá, 19.II.1943, fl. e fr., R.L. Froés 20141 (holótipo IAN!, isótipos F [imagem!], K [imagem!]).

Phthirusa caatingae Rizzini, Revista Fac. Agron. (Maracay) 8(3): 90. 1975. - Tipo: Brasil. Bahia: rodovia BR - 4, a $300 \mathrm{~km}$ de Salvador para Jequié, Caatinga, beira de estrada, 28.I.1965, fl. e fr., R.P. Belém \& J.M. Mendes 325 (holótipo RB 176228 [imagem!], isótipos IAN!, UB!, RB 130774!). Phthirusa trichodes Rizzini, Revista Fac. Agron. (Maracay) 8(3): 92. 1975. Pusillanthus trichodes (Rizzini) Kuijt, Novon 18(3): 372, figs. 1-2. 2008. - Tipo: Venezuela. Lara: Distr. Palavecino, bosque tropofilo seco enano, laderas pendientes entre Terepaima y Cabudare, mirando al noroeste, 500 - 900 m, 5-10.VIII.1970, fl. e fr., J.A. Steyermark et al. 103648 (holótipo RB [imagem!], isótipos F [imagem!], MO [imagem!], VEN [imagem!]).

O holótipo J. Saer 472 depositado no herbário VEN-800, infelizmente não foi localizado pela curadoria do referido herbário. Porém um isótipo foi encontrado no acervo do herbário $\mathrm{F}$, e segundo o Artigo 9.12 do Código Internacional de Nomenclatura Botânica (McNeill et al. 2012), este tem prioridade na lectotipificação sobre o parátipo. Desta forma estamos aqui propondo a lectotipificação deste material.

Além disso, registrou-se novos hospedeiros para a espécie, a saber: Malvaceae (Viana 2104), Pilocarpus racemosus Vahl (Rutaceae - Pittier 13102) e Nicotiana glauca Graham (Solanaceae - Pickel 3917).

Parátipos: Phthirusa trichodes: Brasil. Paraíba: Areia Branca, 14.X.1953, J.C. Moraes 1011 (RB [imagem!]). Struthanthus pubescens: Venezuela. lara: road El Tocuyo - Humocaro Bajo, on n ${ }^{\circ} 13102$, 06.I.1929, fl. e fr., H.F. Pittier 13103 (F [imagem!], MO [imagem!]).

Novos materiais examinados: BRASIL. BAHIA: Paulo Afonso, Raso da Catarina, sobre Malvaceae (Viana 2104), 18.VI.1988, G. Viana 2116 (ASE!). PERNAMBUCO: Araruna, epiphytic upon Nicotiana glauca, 29.VI.1935, fl., D.B. Pickel 3917 (F [imagem!]).

Struthanthus meridionalis Kuijt, Bot. J. Linn. Soc. 142: 469, figs. 1-2. 2003. - Tipo: Bolívia. Tarija. Prov. Arce [Aniceto Arce], hillsides vicinity of Sidras, (5.5 km N of Emborozú), 22 ${ }^{\circ} 12^{\prime} \mathrm{S}, 64^{\circ} 32^{\prime} \mathrm{W}$, $950 \mathrm{~m}$, on legume (Solomon 11138), 9-11.X.1983, J.C. Solomon 11148 (holótipo MO; isótipos LPB [imagem!], MG!, UC).

Struthanthus polyanthus (Mart.) Mart. var. gracilis Rizzini, Arq. Jard. Bot. Rio de Janeiro 24: 22, fig. 2.1. 1980. - Tipo: Brasil. Mato Grosso. Serra do Roncador, varzea forest beside Rio Sete Septembro, $3 \mathrm{~km}$ from Garapu, on Moriria, 02.X.1964, fl., G.T. Prance et al. 59232 (holótipo RB [imagem!]; isótipos MO, UB!), syn. nov.

Analisando o acervo dos herbários MG e UB descobriu-se que, apesar de terem sido registradas em países diferentes (ca. $1600 \mathrm{~km}$ de distância), Struthanthus meridionalis Kuijt, recentemente descrita para a Bolívia, corresponde a S. polyanthus var. gracilis Rizzini, registrada para o Brasil.

De acordo com o Artigo 11.2 do Código Internacional de Nomenclatura Botânica (McNeill et al. 2012), o princípio da prioridade só é utilizando dentro de um mesmo nível de categoria taxonômica e, portanto, modificações nomenclaturais não são necessárias.

Rizzini (1980) não indicou o herbário onde o holótipo de Struthanthus polyanthus (Mart.) Mart. 
var. gracilis Rizzini está depositado, porém o nome foi validamente publicado, pois segundo o Art. 40.7 do Código Internacional de Nomenclatura (McNeill et al.2012), somente a partir de 1990 a indicação do herbário depositário do holótipo seria necessária. Como não há anotações do autor nos isótipos localizados no MO e no UB, o holótipo, portanto, é o material depositado no RB, a instituição na qual trabalhava Carlos Toledo Rizzini.

Rizzini (1980) discute a semelhança de S. polyanthus var. gracilis com $S$. angustifolius (Griseb.) Hauman, cuja diferença seria ausência de estaminódios nas flores pistiladas observadas na primeira. Kuijt (2003) relaciona S. meridionalis com $S$. prancei Kuijt, os quais apresentam uma diversidade na combinação de mônades, díades e tríades em suas inflorescências, incomuns em Struthanthus, mas não suficientes para posicionálas em outro gênero.

Nas análises, observou-se que a morfologia foliar (ápice, textura e venação) juntamente com a densa ramificação acompanhada de inúmeras inflorescências laxas e delicadas individualizam S. meridionalis.

Para essa espécie registramos como hospedeiro: Parapiptadenia excelsa (Griseb.) Burkart (Solomon 11138 - Fabaceae).

\section{Agradecimentos}

Aos digitalizadores dos espécimes, especialmente a Erika Medeiros (RB) e Lauren Peters (MO). Aos curadores dos herbários, principalmente MG, MO, RB e UB, por disponibilizarem os seus acervos para o estudo. Ao Prof. Dr. Jefferson Prado, as dicas sobre nomenclatura. Esta é a publicação 13 da série técnica do Parasitic Plants Research Group.

\section{Referências}

Arruda, R.; Fadini, R.F.; Carvalho, L.N.; Del-Claro, K.; Mourão, F.A.; Jacobi, C.M.; Teodoro, G.S.; van den Berg, E.; Caires, C.S. \& Dettke, G.A. 2012. Ecology of Neotropical mistletoes: an important canopydwelling component of Brazilian ecosystems. Acta Botanica Brasilica 26: 264-274.

Caires, C.S.; Gomes-Bezerra, K.M. \& Proença, C.E.B. 2012. Novos sinônimos e uma nova combinação em Pusillanthus (Loranthaceae). Acta Botanica Brasilica 26: 675-681.

Caires, C.S.; Gomes-Bezerra, K.M. \& Proença, C.E.B. 2014. A new combination in Peristethium
(Loranthaceae) expands the genus' range into the Amazon-Cerrado ecotone. Acta Amazonica 44: 169-173.

Dettke, G.A. \& Waechter, J.L. 2012. Struthanthus martianus, a replacement name for the illegitimate Struthanthus vulgaris Mart. ex Eichler (Loranthaceae). Phytotaxa 57: 6-9.

Kuijt, J. 2003. Two new South American species of Struthanthus (Loranthaceae) posing a challenge to circumscription of neotropical genera. Botanical Journal of the Linnean Society 142: 469-474.

Kuijt, J. 2008. Pusillanthus (Loranthaceae), new monotypic genus from Venezuela. Novon 18: 370-373.

Kuijt, J. 2011. Pulling the skeleton out of the closet: resurrection of Phthirusa sensu Martius and consequent revival of Passovia (Loranthaceae). Plant Diversity and Evolution 129: 159-211.

Kuijt, J. 2012. Reinstatement and expansion of the genus Peristethium (Loranthaceae). Annals of the Missouri Botanical Garden 98: 542-577.

Kuijt, J. 2013. A brief taxonomic history of neotropical mistletoe genera, with a key to the genera. Blumea 58: 263-266.

Kuijt, J. 2014a. Five new species, one new name, and transfers in Neotropical mistletoes (Loranthaceae), Miscellaneous Notes, 61-68. Novon 23: 176-186.

Kuijt, J. 2014b. Lectotypification of Loranthus thyrsiflorus (Loranthaceae) and its placement in Struthanthus. Phytotaxa 181: 120-120.

McNeill, J.; Barrie, F.R.; Buck, W.R.; Demoulin, V.; Greuter, W.; Hawksworth, D.L.; Herendeen, P.S.; Knapp, S.; Marhold, K.; Prado, J.; Prud'Homme van Reine, W.F.; Smith, G.F.; Wiersema, J.H. \& Turland, N.J. (eds.). 2012. International Code of Nomenclature (Melbourne Code) for the algae, fungi, and plants: adopted by the Eightheenth International Botanical Congress Melbourne, Australia, July 2011. Regnum Vegetabile. Vol. 154. Koeltz Scientific Books, Koenigstein. 240p.

Rizzini, C.T. 1950. Struthanthi brasiliae eiusque vicinorum. Revista Brasileira de Biologia 10: 393-408.

Rizzini, C.T. 1975. Loranthaceae novae. Revista de la Facultad de Agronomia (Maracay) 8: 83-109.

Rizzini, C.T. 1980. Loranthaceae of the Central Brazil. Arquivos do Jardim Botânico do Rio de Janeiro 24: $19-50$.

Thiers, B. 2014. Index herbariorum: a global directory of public herbaria and associated staff. New York Botanical Garden's Virtual Herbarium. Disponível em $<$ http://sweetgum.nybg.org/ih/>. Acesso em 11 julho 2014. 